\title{
MODELO DE ATUALIZAÇÃO DE BASES DE CONHECIMENTO: um estudo de caso ONTO-AmazonTimber
}

Knowledge base update model: an ONTO-AmazonTimber case study

\author{
Ademilson de Almeida Barbosa ${ }^{1}$, Márcio José Moutinho Da Ponte ${ }^{2}$, Celson Pantoja Lima ${ }^{3}$ \\ (1) Universidade Federal Do Oeste Do Pará / Instituto De Engenharia E Geociências \\ Programa De Computação, Av. Mendonça Furtado, 2946 - Fátima, Campus Universitário \\ Santarém - PA, 68040-470, ad.ufopa@gmail.com. \\ (2) Universidade Federal Do Oeste Do Pará / Instituto De Engenharia E Geociências \\ Programa De Computação, Av. Mendonça Furtado, 2946 - Fátima, Campus Universitário \\ Santarém - PA, 68040-470, marcio.ponte@ufopa.edu.br. \\ (3) Universidade Federal Do Oeste Do Pará / Instituto De Engenharia E Geociências \\ Programa De Computação, Av. Mendonça Furtado, 2946 - Fátima, Campus Universitário \\ Santarém - PA, 68040-470, celson.lima@ufopa.edu.br.
}

\begin{abstract}
Resumo:
O contexto tecnológico apresenta novos desafios ao processo de produção e atualização de conhecimento. A constante manutenção de bases de conhecimento, permite a evolução no domínio do conhecimento, no entanto somente a consistência do conhecimento inserido, possibilita a eficácia dos resultados nas interações semânticas. Neste contexto, este trabalho objetiva desenvolver um modelo de atualização de bases de conhecimento, e será validado por um estudo de caso aplicado a ontologia ONTO-AmazonTimber, desenvolvida como suporte ao processo de identificação botânica em espécies florestais da Amazônia. O desenvolvimento da pesquisa, utiliza-se da metodologia e-COGNOS e o método de atualização de bases conhecimento, que obedece três processos: o cadastro e a validação do conhecimento e, a inserção deste na base de conhecimento. Com a aplicação do modelo de atualização na ontologia ONTO-AmazonTimber, verificou-se que é possível minimizar a inserção de conhecimentos redundantes e incorretos e, a consistência do conhecimento atribuído que repercute em toda a estrutura semântica.
\end{abstract}

Palavras-chave: Gestão do Conhecimento; Ontologia; Atualização de conhecimento; ONTOAmazonTimber.

\begin{abstract}
:
The technological context presents new challenges to the process of production and updating of knowledge. The constant maintenance of knowledge bases, allows the evolution in the domain of knowledge, however only the consistency of the inserted knowledge, makes possible the effectiveness of the results in the semantic interactions. In this context, this work aims to develop a knowledge base updating model, and will be validated by a case study applied to the ONTO-AmazonTimber ontology, developed as a support to the botanical identification process in Amazonian forest species. The development of the research uses the e-COGNOS methodology and the knowledge base updating method, which obeys three processes: the registration and validation of knowledge and its insertion into the knowledge base. With the application of the update model in the ONTO-AmazonTimber ontology, it was verified that it is possible to minimize the insertion of redundant and incorrect knowledge and the consistency of the attributed knowledge that has repercussions throughout the semantic structure
\end{abstract}

Keywords: Knowledge management; Ontology; Knowledge Update; ONTO-AmazonTimber.

\section{Introdução}

Segundo Luckesi e Passos (1996) o

conhecimento é o resultado do processamento analítico de informações que fornecem os subsídios essenciais para tomadas de decisões. A Gestão do Conhecimento (GC) por sua vez, está intimamente relacionada ao fator sucesso no processo de tomada de decisões, o que tende a aumentar à medida que se intensifica a interação entre a produção de conhecimento e a tecnologia (ROSSETTI e MORALES, 2007).
Nesta perspectiva, o atual contexto tecnológico direciona-se a produção do conhecimento, o que permite expandir o alcance nos domínios de aplicação e potencializar as funcionalidades de um referencial semântico.

Ocorre, que domínios de conhecimento raramente são estáticos e, assim sendo, a ontologia deve acompanhar a evolução do domínio de conhecimento. Caso mudanças do domínio do conhecimento não sejam mapeadas e incorporadas nas bases de conhecimento, está se tornará estagnada, 
ultrapassada, ineficaz e possivelmente incorreta (PONTE, 2017).

A atualização de bases de conhecimento, pode ser consequência da descoberta de erros na modelagem, ou um reparo no conhecimento de domínio. Para que a aquisição de novos conhecimentos sejam adicionadas, segundo (LÖSCH et.al., 2009), as pesquisas devem ser dedicadas empregando técnicas de revisão de crenças, aquisição de conhecimento, aprendizagem de ontologia e evolução de ontologia para citar apenas algumas. Em um nível mais geral, as especificações de atualização, permitem codificar o conhecimento do processo e associá-lo à ontologia, de modo que possa ser usado para atualizações.

É necessário, nesta senda, que as mudanças mediadas por especificações da atualização de ontologias possam ser diretamente evidentes para o construtor do conhecimento (LÖSCH et. al., 2009). Este, por sua vez é crucial para garantir que o comportamento do sistema seja totalmente transparente ao construtor desse conhecimento

No que se refere ao contexto semântico, o excesso ou escassez da abordagem do domínio pode torna-se volumoso ou obsoleto. Para Prado (2001), segundo os critérios para construção de uma base de conhecimento, toda a aquisição de conhecimento é destacada como uma prioridade e, todo o conhecimento deve ser validado por especialistas que consigam destacar a relevância do conhecimento para a base de domínio da ontologia.

\section{Objetivos}

Este trabalho visa propor um modelo de atualização para bases de conhecimento, e como estudo de caso instancia-se o modelo de atualização para a base de conhecimento da ontologia ONTO-AmazonTimber.

\section{Procedimentos Metodológicos}

Este tópico destina-se a apresentar as delimitações metodológicas deste trabalho, isto inclui as metodologias aplicadas, os métodos e procedimentos utilizados para o desenvolvimento do modelo de atualização de bases de conhecimento.
3.1 Metodologia para o desenvolvimento da Ontologia

A metodologia aplicada neste trabalho advém do projeto e-COGNOS (Lima, ElDiraby e Stephens, 2005) no qual foi desenvolvida uma plataforma de gestão do conhecimento baseada na web.

O método proposto para o desenvolvimento da ontologia, foi inspirado na abordagem usada pelo projeto eCOGNOS, motivado pela participação da construção e refinamento da metodologia e do histórico de bons resultados angariados até o momento.

Os principais conceitos que servem como a espinha dorsal da ontologia também foram inspirados na ontologia e-COGNOS. No entanto, para o propósito deste artigo, algumas adaptações e refinamentos do modelo ontológico tiveram de ser feitas.

O método aqui adotado usa uma abordagem iterativa (Figura 01), que é dividida em várias fases, com cada fase contendo um conjunto de tarefas relacionadas. $^{1}$

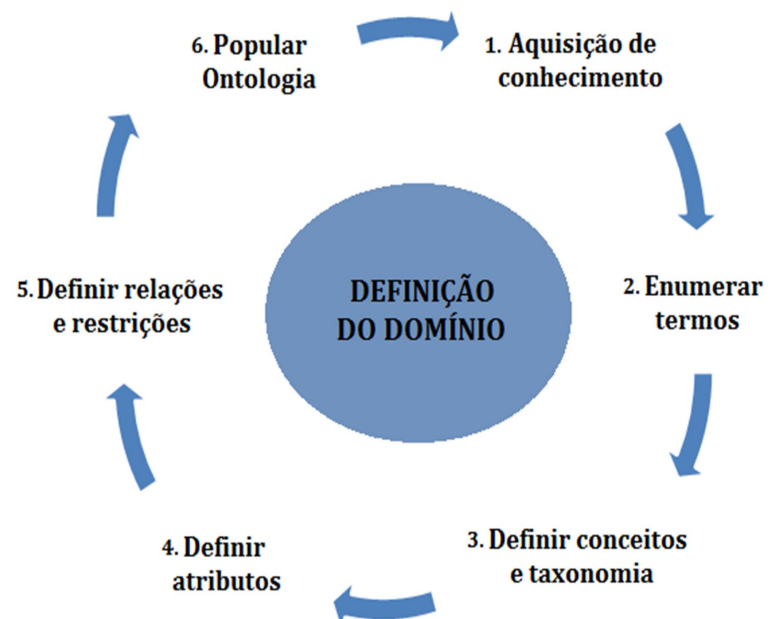

Figura 01. Metodologia e-COGNOS para construção da Ontologia, fonte: Adaptado de Costa (2014).

\subsection{Inserção na estrutura semântica da Ontologia}

O método de atualização para a base de conhecimento proposto, se utiliza de uma ontologia de referência, nomeada como ontologia ONTO-AmazonTimber, para assim conseguir adicionar novos conceitos a ontologia básica.

\footnotetext{
${ }^{1}$ Para uma descrição mais detalhada da metodologia empregada analisar em PONTE, 2017.
} 
Este método é realizado seguindo três etapas. Em primeiro lugar se utiliza um medida de similaridade semântica na ontologia de referência, afim de reconhecer ou encontrar o sentido mais similar ao conceito, de modo a adicionar a ontologia básica.

$\mathrm{Na}$ segunda etapa, se procura a posição correta em que este conceito condiz com o sentido e pode ser encaixado a ontologia básica. No fim o conceito é adicionado a ontologia básica sempre respeitando a estrutura hierárquica.

\section{Resultados}

A ONTO-AmazonTimber trata-se de um referencial semântico no âmbito da botânica que formaliza o conhecimento do especialista (taxonomista) na tarefa de identificação botânica. Dispondo de conhecimentos como: caracterização das espécies botânicas comercializadas no setor madeireiro da Amazônia; caracterização do ambiente em que estão inseridas; e caracterização do contexto ambiental.

Para validar o modelo apresentado nesse trabalho, introduziu-se uma abordagem de resultados baseados em uma proposta de modelo de atualização de bases de conhecimento a partir do estudo de caso da ontologia ONTO-AmazonTimber.

4.1 Modelo conceitual de atualização de base de conhecimento

Segundo Lisboa (2000), os modelos conceituais permitem representar de maneira abstrata, formal e não ambígua a realidade da aplicação, com objetivo de facilitar a comunicação entre os envolvidos no projeto.

O modelo conceitual apresentado neste trabalho, permite mensurar o escopo do planejamento de atualização da base de conhecimento, assim como a base teórica do domínio do conhecimento e a especificação da estruturação dos elementos envolvidos, possibilitando que um novo conhecimento possa compor a base de conhecimento.

Para que haja sustentação do modelo conceitual proposto, os seguintes elementos estruturais são fundamentais: Especialista, Conhecimento, Comissão de Validação e Base de Conhecimento (Figura 02).
O Especialista representa um grupo de profissionais que trabalham no domínio do conhecimento da problemática em questão. Sua formação e experiência são consideradas de alto valor quando se trata da formalização e atualização do conhecimento.

A comissão de validação se utiliza dos seguintes critérios: a Formação e, a Experiência do Especialista quando avalia a proposta de um novo conhecimento para ser inserido a base de conhecimento.

Note-se, que nesta etapa o conhecimento ainda não foi inserido, tendo em vista a atuação do especialista que no processo de validação, confere ao conhecimento mera expectativa de inserção, já que somente quando um novo conhecimento é validado, que este irá compor a base de conhecimento.

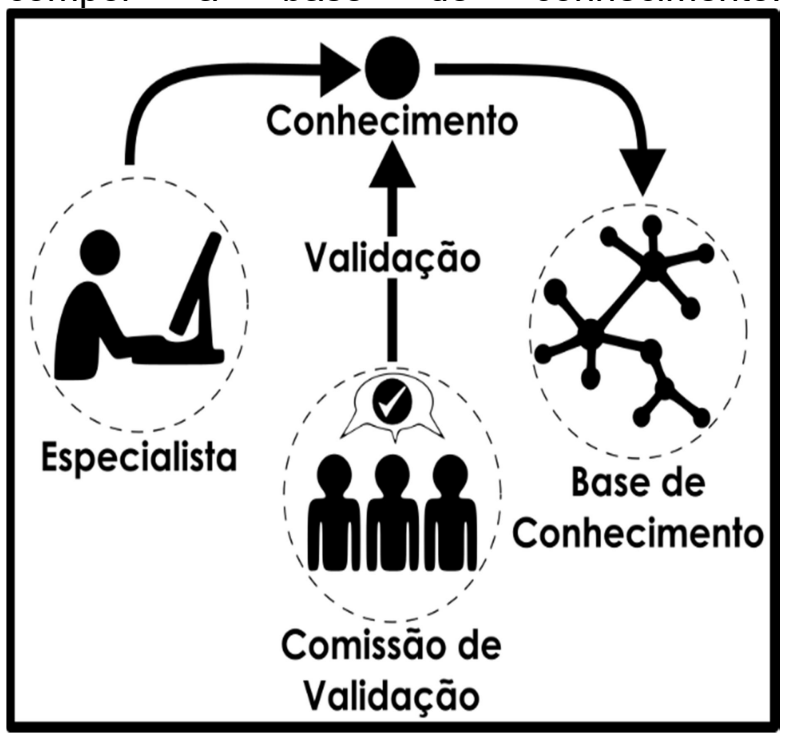

Figura 02. Modelo de Atualização, fonte: dados da pesquisa, (2017).

4.2 Instanciação do Modelo de atualização aplicado a ONTO-AmazonTimber.

A instanciação do modelo de atualização desenvolvido neste trabalho é representada por elementos estruturais que se fundamentam quando aplicados no âmbito da botânica (Figura 03).

O Especialista representa um grupo de profissionais que atuam no processo de identificação botânica, destacam-se: Taxonomistas, Parataxonomista, Botânicos e Engenheiros Florestais com experiência em inventário florestal. As especializações, habilidades, experiências e formações no domínio da botânica servem como quesitos 
fundamentais na formalização de novos conhecimentos.

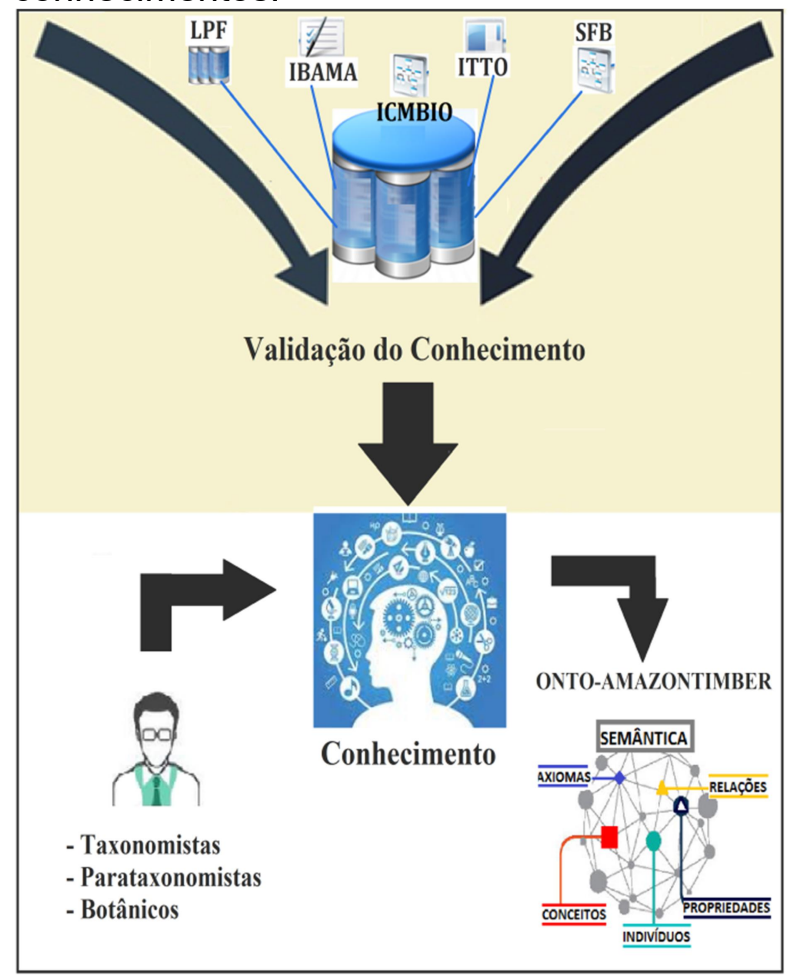

Figura 03. Instanciação do Modelo de Atualização, fonte: dados da pesquisa, (2017).

A comissão de validação é representada por indivíduos inseridos em entidades de fiscalização, pesquisa e promoção da sustentabilidade ambiental, a citar: Instituto Brasileiro do Meio Ambiente e dos Recursos Naturais Renováveis (IBAMA), Laboratório de Produtos Florestais (LPF) da Universidade Federal do Oeste do Pará (UFOPA), Serviço Florestal Brasileiro (SFB), Instituto Chico Mendes de Conservação da Biodiversidade (ICMBio).

O modelo de atualização proposto apresenta os seguintes processos: o especialista propõe um novo conhecimento. Tal conhecimento é avaliado pela comissão de validação, que devem utilizar os seguintes critérios de avaliação: formação e experiência do profissional, para assim analisar e validar o novo conhecimento.

A base de conhecimento da ONTOAmazonTimber trata-se do terceiro elemento do modelo, formalizado por uma estrutura semântica composta por conceitos, relações, propriedades e axiomas, instanciados e interrelacionados para compor o domínio de conhecimento em questão.
4.3 Interfaces do modelo de atualização da ONTO-AmazonTimber

Neste tópico apresentam-se protótipos de telas desenvolvidos na elaboração do modelo de atualização. $O$ processo de interação do modelo de atualização inicia-se com a seleção da espécie de interesse (Figura 04).

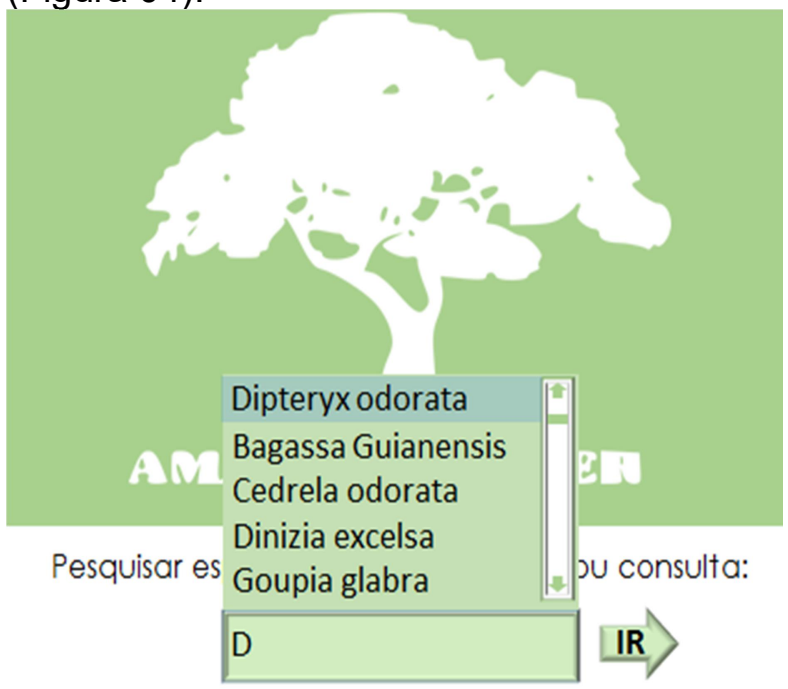

Figura 04. Tela de seleção, fonte: dados da pesquisa, (2017)

Posteriormente é exibido todas as relações semânticas de propiciam a caracterização da espécie selecionada (Figura 05). Permitindo desta forma incluir uma nova características.

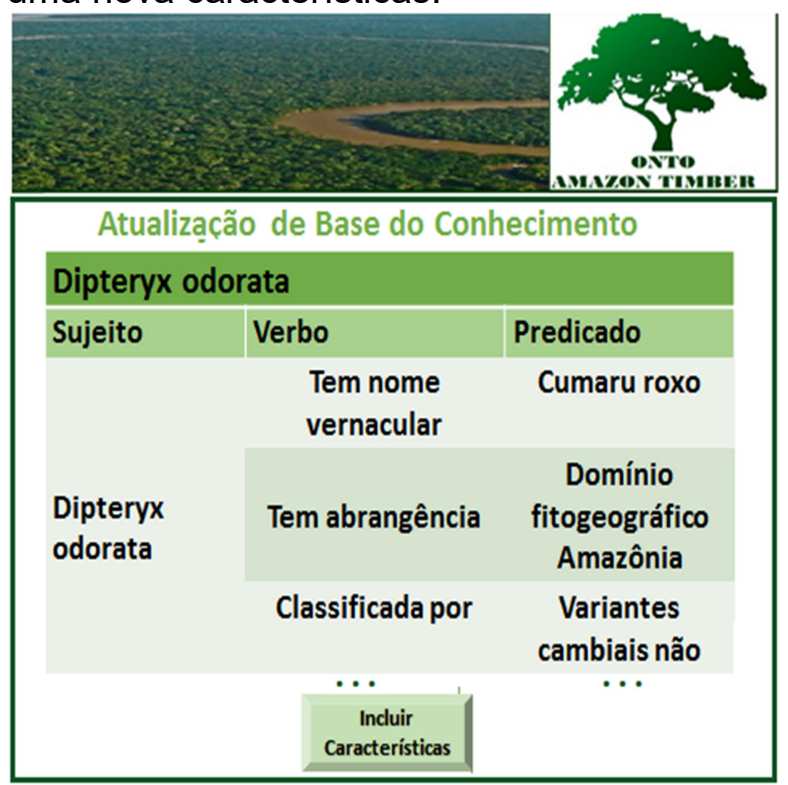

Figura 05. Tela de caracterização, fonte: dados da pesquisa, (2017). 
Desta forma na tela de inclusão (Figura 06), é selecionado a estrutura semântica e incluir ou cadastrar novas espécies, verbos e predicados.

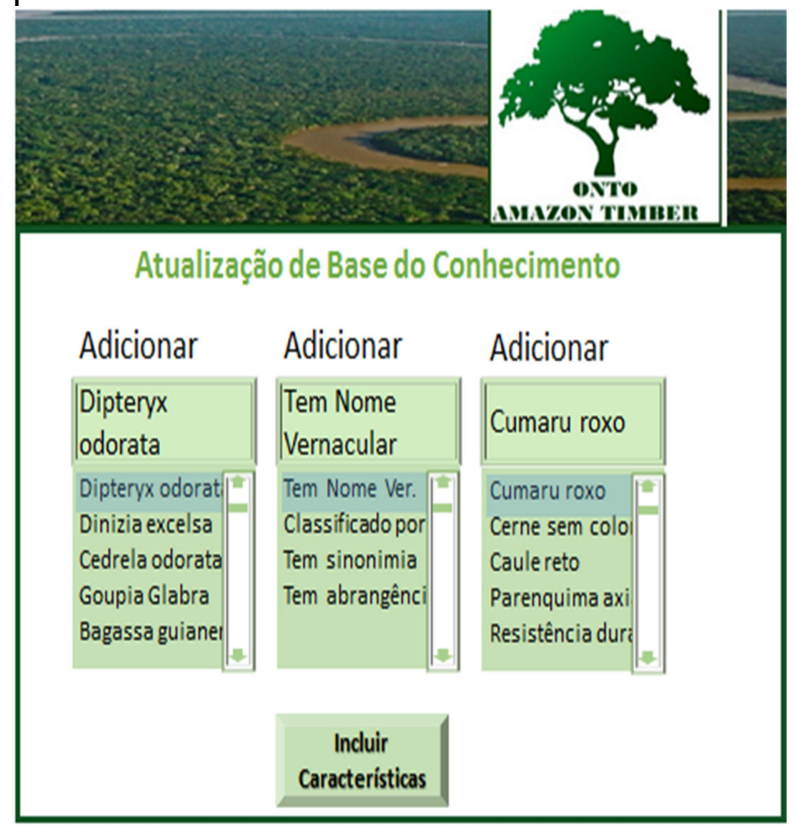

Figura 06. Tela de inclusão, fonte: dados da pesquisa, (2017).

\subsection{Interação Ontológica com JENA}

A JENA consiste em um framework JAVA que permite trabalhar em ambiente de programação com manipulação dinâmica de modelos RDF (Resource Description Framework), representadas pelos recursos, propriedades e literals, formando as tuplas (predicate, [subject], [object]) que originam os objetos criados pelo JAVA.

Este Framework disponibiliza um kit de funcionalidades para apoiar 0 desenvolvimento de aplicações no contexto de ontologias. Além das funcionalidades para manipulação da linguagem OWL e uso do Simple Protocol And Rdf Query Language (SPARQL).

A API JENA apresenta um conjunto de métodos que permitem acessar os elementos de uma Ontologia (classes, propriedades e indivíduos) podemos utilizar os métodos iniciados com list como listClasses(), listIndividuals() ou listSubClasses(). A partir deles pode-se chamar o método toList() para ter acesso aos elementos através de uma instância da classe java.util.List. Além disso, para identificar qual classe ou instância está sendo manipulada dentro das iterações, temos dois métodos básicos: getURI(), que retorna o nome completo ou a URI (prefixo + nome) do objeto; e getLocalName(), que retorna apenas o nome do objeto em questão.

Outros métodos permitem uma maior especificação quanto ao acesso a estrutura ontológica, $\quad 0 \quad$ método getObjectsFromObjectTriple possibilita listar um conjunto de objetos da classe $A$ que se relacionam através de uma propriedade específica com um outro objeto da classe $B$. Para melhor entendimento visualiza-se o código abaixo:

OntologyInteraction listaCaracteristica = new OntologyInteraction() ;

$$
\begin{aligned}
& \text { ArrayList<String> objects } \\
& \text { listaCaracteristica. } \\
& \text { getObjectsFromObjectTriple("Dipteryx_odo } \\
& \text { rata", "ClassificadoPor"); }
\end{aligned}
$$

Tal método getObjectsFromObjectTriple tem como função listar as características botânicas da espécie botânica Dipteryx_odorata que estão interligadas pela propriedade ClassificadaPor, tal relação semânticas podem ser observadas na (Figura 07), no qual a classe Species tem uma série de objetos dentre estes 0 Dipteryx_odorata, por sua vez apresenta algumas propriedade de objetos que criam relações com outros objetos, como por exemplo: Heartwood_Distinct_Color instancia da classe Heartwood_Color. 


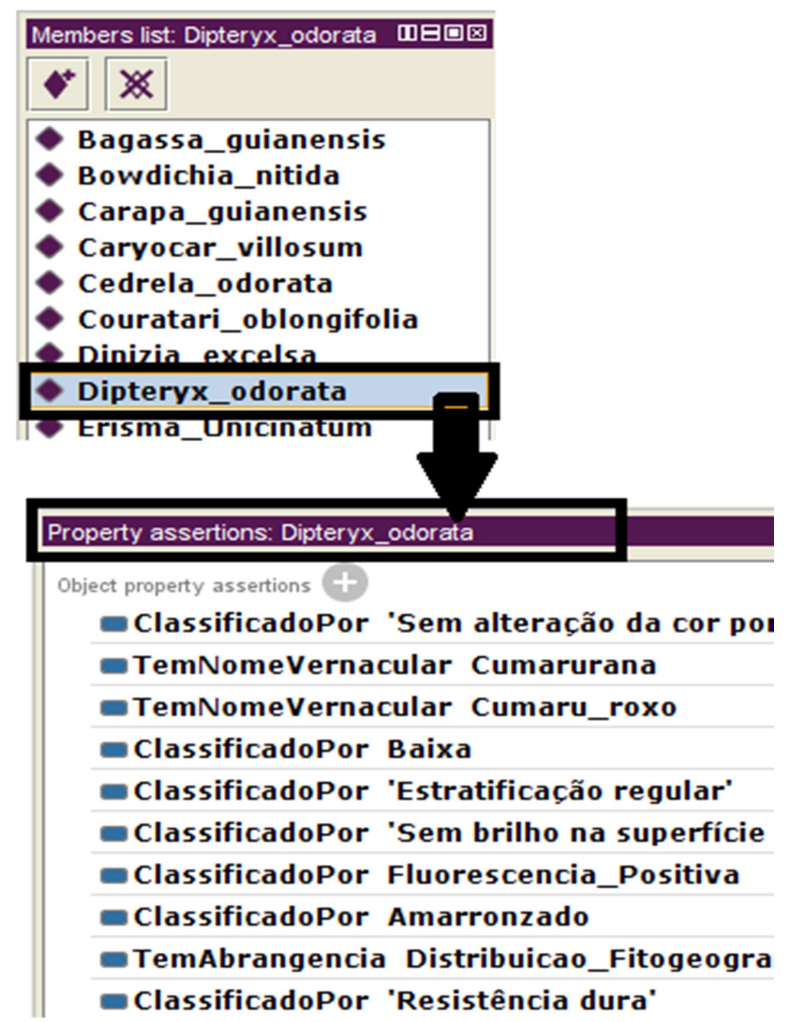

Figura 07. Relações semânticas obtidas pelo método getObjectsFromObjectTriple, fonte: PONTE (2017).

\section{Considerações Finais}

O modelo conceitual de atualização desenvolvido neste trabalho apresenta requisitos necessários para manutenção de bases do conhecimento nos mais diversos domínios do conhecimento. Premissa necessária para contínua evolução da estrutura semântica, item indispensável na Gestão do Conhecimento.

Considerando-se a extensa diversidade biológica da Amazônia e a vasta quantidade de espécies florestais existentes na Amazônia, aliado a escassez de profissionais que atuem no processo de identificação botânica, evidencia-se a extrema necessidade da constante atualização da base de conhecimento.

O modelo de atualização proposto permite minimizar a inserção de conhecimentos redundantes e incorretos e, a consistência do conhecimento atribuído que repercute em toda a estrutura semântica.

\section{Referências}

COSTA, R. D. D. Semantic Enrichment of Knowledge Sources Supported by Domain
Ontologies. Tese de Doutorado em Engenharia Electrotécnica e de Computadores - Faculdade Ciências e Tecnologia, Universidade Nova de Lisboa, Portugal - Lisboa, 2014.

LIMA,C.; EL-DIRABY,T.; STEPHENS,J. Ontology-basedoptimisation of knowledge management in e-Construction. ITcon 10, 305-327. 2005.

LISBOA F., J. Modelagem de Banco de Dados Geográficos. In: LADEIRA, M.; NASCIMENTO, M. E. M. III Escola Regional de Informática do Centro-Oeste. Brasília - DF. SBC - Sociedade Brasileira de Computação, 2000.

LÖSCH U., RUDOLPH S., VRANDE`CI'C D., and STUDER R., "Tempus fugit: Towards an Ontology Update Language", Institut AIFB - Universit"at Karlsruhe (TH): Karlsruhe, Germany: 2009.

LUCKESI, C. C. e PASSOS, E.S. "Introdução à filosofia: aprendendo a pensar.", São Paulo: Cortez, 1996.

MOTA, M. R. A. "Mapeamento Sistemático Sobre o Uso de Ontologias em informática Médica", João Pessoa: Agosto, 2013.

PONTE M. J. M, "Referencial Semântico no suporte da identificação botânica de Espécies Amazônicas", Tese de doutorado - UFOPA/UNL -Santarém Pará, Abril 2017.

PRADO, E. V. "Sistema Especialista para dimensionamento e seleção de equipamentos para pré-processamento de café ll", "Tese de doutorado" - UFV, 2001.

ROSSETTI A. G., MORALES A. B. T. O papela da tecnologia da informação na gestão do conhecimento. C i. Inf., Brasília, v. 36, n. 1, p. 124-135, jan./abr. 2007.

RUSSEL, S. N. P. "Artificial Intelligence: A Modern Approach." 3rd Edition, New Jersey: Prentice Hall; 2009. 